\title{
Clinical and Radiographic Comparison of Oxidized Zirconium and Cobalt Chrome Femoral Components of a Single Design Primary Total Knee Arthroplasty: Is the Cost Difference Justified Based on Patient Outcomes?
}

\author{
Geevan George, ${ }^{1}$ Nicole Durig, ${ }^{2}$ Stewart Lee, ${ }^{1}$ Stephanie Tanner, ${ }^{3}$ \\ Rebecca Snider, ${ }^{3}$ and Thomas Pace ${ }^{3}$ \\ ${ }^{1}$ University of South Carolina School of Medicine Greenville, 701 Grove Road, Greenville, SC 29605, USA \\ ${ }^{2}$ Clemson University Biomedical Engineering Innovation Campus, 200 Patewood Drive, Greenville, SC 29615, USA \\ ${ }^{3}$ Greenville Health System, Department of Orthopedics, University of South Carolina SOM, 2nd Floor Support Tower, \\ 701 Grove Road, P.O. Box 27114, Greenville, SC 29616, USA
}

Correspondence should be addressed to Thomas Pace; tpacemd@aol.com

Received 20 August 2013; Accepted 20 October 2013

Academic Editors: V. K. Balla, E. Gómez-Barrena, and B. Yang

Copyright (C) 2013 Geevan George et al. This is an open access article distributed under the Creative Commons Attribution License, which permits unrestricted use, distribution, and reproduction in any medium, provided the original work is properly cited.

A review of 120 consecutive total knee arthroplasty patient records was made comparing oxidized zirconium with traditional cobalt chrome alloy femoral components and assessing the clinical and radiographic outcomes of each. The direct hospital implant cost for each of these implant systems was obtained and assessed in the context of any difference in patient Knee Society Scores, knee flexion, pain, probability of metal allergy, and radiographic signs of pending implant failures. At an average of 5.6-year followup (111-year range in both groups), the data showed no clinical or radiological variance between the two groups, while the hospital direct implant cost with the oxidized zirconium femoral component was $\$ 1900$ higher than the cobalt chrome implants. Considering the cost difference between the implants and the lack of a difference in outcomes at an average of 5-6 years of followup, this paper brings into question the ability to justify the use of oxidized zirconium femoral components based solely on improved patient outcomes.

\section{Introduction}

The volume of joint arthroplasty procedures performed each year has steadily increased over time, with primary total knee arthroplasty (TKA) projected to increase by $673 \%$, from 450,000 in 2005 to 3.48 million procedures in 2030 [1]. With the increased population of active and aging baby-boomers qualifying for Medicare and the changes to the health care system implemented by the Affordable Care Act, providers and policy makers must make decisions on procedures and products that are both clinically effective and cost efficient. Advances in TKA technology have introduced new materials aimed at providing quicker recovery, hypoallergenic properties, and improved prosthesis survivorship.

Oxidized zirconium is a relatively new material consisting of a zirconium alloy $(\mathrm{Zr}-2.5 \mathrm{Nb})$ core that has been oxidized to produce a zirconia $\left(\mathrm{ZrO}_{2}\right)$ ceramic-like surface. Knee simulator studies have demonstrated the proposed benefits of oxidized zirconium, which include less nickel exposure and improved wear properties, such as increased hardness, decreased surface roughness, and increased resistance to abrasion, adhesion, and delamination compared to cobaltchromium-molybdenum (CoCrMo) [2-9]. Several in vivo studies have been published concerning the clinical effectiveness and wear performance of oxidized zirconium femoral components $[7,10,11]$; however, many have reported similar clinical outcomes for the oxidized zirconium in comparison to the traditional CoCrMo alloy [12-15]. Since oxidized zirconium requires a more complex manufacturing process, including undergoing a high-temperature oxidation treatment [3], these prosthesis components are more expensive than those composed of traditional metal alloys. With anticipated limitations on resources in the healthcare system in the future, providers and policy makers would justifiably need to 
know if there is a difference in clinical effectiveness of the different biomaterials, in order to justify the increased costs of those that are more advanced.

The present study assesses clinical and radiographic outcomes in a consecutive series of osteoarthritic knee patients comparing the use of a single primary TKA design with femoral components fabricated from either oxidized zirconium or CoCrMo alloy. Due to the promising results of previously published knee simulator studies $[6,8]$, it is hypothesized that oxidized zirconium femoral components, compared to their traditional CoCrMo alloy counterparts, would demonstrate improved clinical and radiographic outcomes, which may justify the cost difference for the more advanced material.

\section{Materials and Methods}

Between May 2001 and October 2002, 120 consecutive primary TKAs were performed in 118 patients by a single surgeon and coauthor (TBP). In this institutional review board approved study, data was retrospectively collected from each patient's pre- and annual postoperative clinical assessments. Sixteen patients were lost to death or did not achieve the minimum follow-up time of 1 year, providing a final cohort of 104 knee surgeries in 102 patients.

All patients were implanted with the same TKA design (Profix Total Knee System, Smith \& Nephew, Memphis, TN, USA). This design provides the option for using one of four different modular tibial central stems; however, all patients in this study received the same short, surface-textured stem (Figure 1). While all of the tibial base plates were fabricated from a titanium alloy, the femoral components were fabricated from a CoCrMo alloy in 84 knees and oxidized zirconium in 20 knees. Femoral components composed of CoCrMo alloy were used in all patients prior to the FDA approval and release of oxidized zirconium TKA implants. Following the release of in vitro studies indicating more favorable wear characteristics for the new material, oxidized zirconium was generally implanted in younger and more active, high-demand patients [8]. All tibial inserts were machined from conventional ultra-high-molecular-weight polyethylene (UHMWPE), rather than highly crosslinked UHMWPE and had been sterilized in ethylene oxide. Additionally, all implanted inserts had an anterior constraint (Conforming Plus, Smith \& Nephew, Memphis, TN, USA). Both femoral and tibial components were fixed using bone cement (Palacos R cement, Biomet Inc., Warsaw, IN, USA) in all but one patient with a self-proclaimed cement (barium) allergy. A surface cementation technique was used to fix the tibial component such that the bone cement was applied to the undersurface of the base plate, excluding the pegs and stem.

The indication for TKA was osteoarthritis for all patients. The PCL was sacrificed in all 104 cases, and a polyethylene insert incorporating an anterior-constrained articular geometry was utilized to provide anteroposterior stability. The medial parapatellar approach was used in all but 3 cases (all CoCrMo alloy cohort), which were exposed using

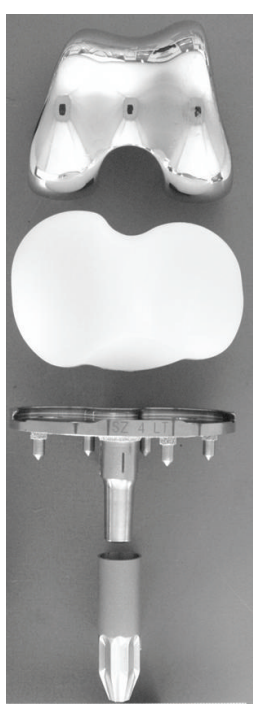

Figure 1: Gross photograph of the Profix TKA (with CoCrMo alloy femoral component).

the subvastus medial approach. The subvastus approach was performed on patients if the distal thigh circumference was small enough that it could be practically done. Patients with a larger distal thigh circumference received the medial parapatellar approach. Ten knees (50\%) in the oxidized zirconium cohort and 33 knees (39\%) in the CoCrMo alloy cohort underwent lateral retinacular release. This procedure was performed based on intraoperative assessment after all prosthesis components were implanted to allow optimal patella tracking. Patella resurfacing was performed in patients if they failed to meet the criteria of having proper congruency of the natural patella, maintaining $85 \%$ of normal appearing cartilage, proper tracking in the trochlear groove, and minimum patellofemoral pain at preoperative assessment. One patient in the CoCrMo cohort underwent patella resurfacing because these criteria were not met.

All patients received a short course of low-molecular weight heparin (Enoxaparin, Sanofi-Aventis, Paris, France) as well as oral warfarin for thromboprophylaxis while hospitalized. Venous thrombosis event (VTE) prophylaxis was $5 \mathrm{mg}$ oral warfarin daily starting the day of surgery with low-molecular-weight heparin injections for post-op days 2 and 3 followed by a 4-week duration of $2 \mathrm{mg}$ per day mini fixed-dose oral warfarin regimen based on individual patient VTE risk assessment. For the patient without higher VTE or bleeding risk assessment, once hospital International Normalized Ratio (INR) levels reached 1.2 or higher, oral warfarin was reduced to $2 \mathrm{mg}$ daily and after discharge INR monitoring was not done unless signs or symptoms of bleeding occurred. For higher-risk VTE patients, higherdose monitored oral warfarin was used (INR target $=2.0$ ). For patients whom postsurgical hemoglobin levels dropped to $8.0-8.5 \mathrm{mg} / \mathrm{dL}$ or lower, transfusion was considered and ensued unless patient requested or other medical conditions prohibited transfusion. Physical therapy was standardized following the hospitals' total joint rehabilitation protocols and included partial weight bearing postoperative day one 
TABLE 1: Demographic data.

\begin{tabular}{lcc}
\hline & Oxidized zirconium & CoCrMo alloy \\
\hline Sample size & 20 & 84 \\
Male $:$ female & $6: 14$ & $20: 64$ \\
Left $:$ right Knee & $9: 11$ & $48: 36$ \\
Age at index surgery & $53 \pm 8(31-65)$ & $69 \pm 10^{*}(44-87)$ \\
$\begin{array}{l}\text { Cost of entire TKA } \\
\text { prosthesis }\end{array}$ & $\$ 5,300$ & $\$ 3,400$ \\
\hline
\end{tabular}

${ }^{*} P$ value less than $0.05(<0.001)$.

and criteria for discharge including independent ambulation, performance of activities of daily living, and passive knee flexion of 80-90 degrees. A continuous passive motion machine was not used after the primary surgery. The same therapy team performed home rehabilitation for two weeks on all patients; this was followed by outpatient therapy.

A range of motion assessment was performed by the operative surgeon at all follow-up appointments and knee flexion was recorded. Patients who failed to obtain 70 degrees of flexion at 2 weeks were treated with anti-inflammatory medications and diuretics. Formal therapy was held and patients worked on knee flexion exercises at home. Radiographic assessment of the surgical knee was performed at the two- or six-week follow-up office visit. The decision to manipulate was made in the 8- to 12 -week period once it was demonstrated that progress in therapy was no longer improving motion, and the patient had less than 90 degrees of flexion.

Manipulations were performed in the operating room under general or spinal anesthesia. After manipulation, patients used continuous passive motion machines at home six hours a day for a total of two weeks. Patients were seen daily by home therapy and had a follow-up visit with the surgeon two weeks after manipulation if it was required.

Patient demographics for knees implanted with femoral components fabricated from both types of materials were comparable (Table 1), including gender and operative side. However, patient age at index surgery was significantly different $(P<0.001)$ between the patient cohorts with the average age being $68 \pm 10$ (44-87) years and $53 \pm 8$ (31-65) years for the CoCrMo alloy group and oxidized zirconium group, respectively.

In order to assess clinical and radiographic outcomes, medical records were retrospectively reviewed. Surgical approach and posterior cruciate ligament (PCL) treatment were recorded at the time of index surgery. Pre- and postoperative Knee Society Scores (KSS) [16] and postoperative maximum flexion were recorded during patient visits. Any pain, complications (including manual manipulation, probable metal allergy, venous thrombotic events (VTE), dehiscence, and infection), or revision surgeries were recorded in subsequent follow-up patient visits. Patients who presented with pain at the most recent follow-up visit were assessed for the possibility of metal allergy. Based on the review of previous reports of metal allergy $[17,18]$, five specific clinical conditions were recorded to assess for possible metal allergy. These were recorded on sequential examinations and included: documented pain, swelling, dermatitis, limited range of motion ( $<115^{\circ}$ flexion), and the prolonged use of pain medications beyond three months following surgery. Using these clinical indications as a guideline, a matrix was used to determine if the patients experiencing pain had a reasonable probability of metal allergy. Patients who presented with pain at the most recent followup and who experienced at least three of the five clinical manifestations were considered to have a reasonable probability of metal allergy.

Radiographic analysis consisted of full-length, standing anteroposterior, sunrise, and lateral view radiographs which were assessed by three independent arthroplasty surgeons for the presence of osteolytic lesions and radiolucent lines greater than $2 \mathrm{~mm}$ located around the femoral and tibial components. In addition to the Knee Society scoring system, poor patient outcomes were defined as probable metal allergy, persistent pain, final flexion of $<90$ degrees, manual manipulation, radiolucencies per serial radiographs, and revision knee surgery.

Quantitative data was analyzed using a two-sample $t$-test. Qualitative data was analyzed using Fisher's exact analysis. Alpha was set at 0.05 .

Implant-specific contract cost for all implants used in this study was obtained from the hospital where the surgeries were performed. The surgical technique and post-op protocol for the two groups differed only by the femoral component materials used. The cost difference was due solely to the difference in cost of the individual femoral components. The purchasing records reflected the hospital's negotiated cost from the manufacturer for both types of prostheses implanted during the time frame in which these TKA were performed. The direct hospital-related implant cost for each type of prostheses can be found in Table 1.

\section{Results}

Clinical outcomes for both the oxidized zirconium and CoCrMo alloy cohorts (including a younger age subgroup within the CoCrMo alloy group) are summarized in Table 2. The average preoperative KSS for oxidized zirconium and CoCrMo alloy prostheses were $76.7 \pm 4.6(70-85)$ and $78.5 \pm$ 4.8 (70-87), respectively. The average postoperative KSS at the most recent follow-up visit were $98.8 \pm 2.8$ (90-100) for oxidized zirconium and $98.9 \pm 2.2$ (90-100) for CoCrMo alloy. The average change in KSS was $22.1 \pm 6.0$ (9-30) for oxidized zirconium and $20.4 \pm 5.1$ (9-30) for CoCrMo. A Two-sample $t$-test was used to analyze the data for KSS and no significant statistical or clinical differences were found between the two materials for preoperative $(P=0.7891)$, postoperative $(P=0.1303)$, or change $(P=0.2781)$ in KSS. A subgroup of the CoCrMo alloy patients that were under the age of 65 years old were also analyzed. In the CoCrMo alloy subgroup, the mean preoperative KSS was $78 \pm 5.0$ (70-86) and postoperative KSS was $98.6 \pm 2.3$ (95-100). The average change in KSS CoCrMo alloy subgroup was $20.6 \pm 4.5$ (1429). When this subgroup of the CoCrMo alloy cohort was compared to the oxidized zirconium cohort, no significant 
TABLE 2: Clinical outcomes.

\begin{tabular}{|c|c|c|c|}
\hline & $\begin{array}{l}\text { Oxidized zirconium } \\
\text { age all }<65(n=20)\end{array}$ & $\begin{array}{l}\text { CoCrMo alloy } \\
\text { all patients }(n=84)\end{array}$ & $\begin{array}{c}\text { CoCrMo alloy } \\
\text { age }<65(n=28)\end{array}$ \\
\hline Followup (years) & $5.2 \pm 3.7(1.0-10.8)$ & $6.0 \pm 3.5(1.0-11.1)$ & $6.7 \pm 4.1(1.0-11.1)$ \\
\hline Preoperative KSS & $76.7 \pm 4.6(70-85)$ & $78.5 \pm 4.8(70-87)$ & $78.0 \pm 5.0(70-86)$ \\
\hline Postoperative Last followup KSS & $98.8 \pm 2.8(90-100)$ & $98.9 \pm 2.2(90-100)$ & $98.6 \pm 2.3(95-100)$ \\
\hline Maximum flexion $\left(^{\circ}\right)$ & $119.3 \pm 3.9(115-128)$ & $116.6 \pm 8.9(60-126)^{*}$ & $116.3 \pm 12.7(60-126)$ \\
\hline $\begin{array}{l}\text { Knees experiencing pain at recent } \\
\text { followup }\end{array}$ & 4 & 8 & 4 \\
\hline Patients with probable metal allergy & 1 & 0 & 0 \\
\hline Knees requiring manual manipulation & 0 & 2 & 2 \\
\hline Knees experiencing VTE & 0 & 0 & 0 \\
\hline Knees requiring revision & 0 & 0 & 0 \\
\hline Knees experiencing other complications & 0 & $2^{* *}$ & $1^{* *}$ \\
\hline
\end{tabular}

TABLE 3: Radiographic outcomes.

\begin{tabular}{lccc}
\hline & Oxidized zirconium $(n=20)$ & CoCrMo alloy $(n=84)$ & CoCrMo alloy, age $\leq 65(n=28)$ \\
\hline Radiolucencies & 0 & 2 & 0 \\
Osteolytic lesions & 0 & 0 & 0 \\
\hline
\end{tabular}

statistical or clinical variations were found for preoperative $(P=0.3596)$, postoperative $(P=0.8139)$, or change $(P=$ 0.3599 ) in KSS.

The summary data on knee flexion (Table 2) reveals the average degrees of maximum flexion measured at the most recent follow-up visit. The average maximum flexion for oxidized zirconium and CoCrMo alloy was $119.3 \pm 3.9$ (115$128)$ and $116.6 \pm 8.9$ (60-126), respectively. A $P$ value of 0.0463 was found, which indicates a statistically significant difference between the two materials. However, a difference of 2.7 degrees is within the expected measurement error of knee flexion and therefore, there was no clinical difference in knee flexion between groups. When comparing the subgroup of CoCrMo alloy (average maximum flexion $116.3 \pm 12.7$ (60-126) degrees) to the oxidized zirconium group, the $P$ value was 0.2584 , demonstrating no statistical difference. The smaller sample size accounts for the difference in assessments of the main CoCrMo group and the younger subgroup despite nearly equal flexion.

Data obtained from patients that clinically presented with pain at their most recent follow-up assessment were also recorded and are shown in Table 2. Four patients with oxidized zirconium prostheses and eight patients with CoCrMo alloy were noted to be experiencing pain $(P=$ 0.2388 ), demonstrating no statistically significant difference. Additionally, four of the twenty-eight patients in the CoCrMo alloy subgroup experienced pain at their most recent followup. When this subgroup was compared to the oxidized zirconium cohort, there was no statistically significant difference between patient groups $(P=1)$.

The records of patients reporting pain at the most recent followup were reviewed for probable metal allergy. The review consisted of the surgeon and coauthor (TBP) assessing the chart of each patient experiencing pain and noting if patients presented with at least three of the five clinical manifestations of a metal allergy defined in previous studies $[17,18]$. Of the patients who experienced pain, only one patient presented with three of the five clinical manifestations of a metal allergy. This patient was implanted with an oxidized zirconium femoral component and had a follow-up period of 3 years.

None of the patients of the present study, in either cohort, required revision surgery for any reason. Additionally, none of the patients included in the oxidized zirconium cohort were required to undergo manual manipulation, experienced a symptomatic VTE, or presented with a complication during follow-up treatment. Two knees, within the CoCrMo alloy cohort, required closed, manual manipulation following implantation. Additionally, two knees in this cohort experienced wound dehiscence two weeks following surgery. Both were treated with secondary wound closure and healed uneventfully. None of the patients in the CoCrMo alloy cohort experienced a symptomatic VTE.

Radiographic outcomes for the different material cohorts are summarized in Table 3 . No radiographs demonstrated the presence of osteolytic lesions in any of the patients. Radiolucent lines adjacent to 2 TKA were noted upon radiographic analysis. Both of these patients received CoCrMo alloy femoral components. In one case, the radiolucent line was determined to be less than $2 \mathrm{~mm}$ in thickness, asymptomatic, and not associated with prosthesis failure. The other case, in which radiographic evidence of a $2 \mathrm{~mm}$ lucent line beneath the tibial tray existed, was further investigated. The patient indicated mild patellofemoral pain, most likely attributed to the unresurfaced patella, and did not require management 
through medication. The radiolucency was not progressive over a two-year follow-up period and was not considered to be associated with failure of fixation of the prosthesis components.

\section{Discussion}

Emerging surgical techniques and biomaterials give patients and surgeons new and innovative tools for improving TKA clinical outcomes and survivorship. Oxidized zirconium is a proprietary material supported as an alternative to traditional CoCrMo alloy due to its hardness, scratch resistance, and hypoallergenic properties in femoral components of TKAs. Knee simulator studies have shown promising results for improved wear properties and biocompatibility of oxidized zirconium compared to traditional metal alloys used to fabricate TKA prostheses [2-9]. The increased hardness and toughness, decreased surface roughness, and increased resistance to specific types of wear of oxidized zirconium should theoretically lower the production of metal and polyethylene particulates being released into the joint capsule, leading to decreased osteolysis and loosening [8]. Thus, one may expect to see decreased clinical and radiographic presentations of wear and metal allergy with oxidized zirconium TKA femoral components. The clinical outcomes of the oxidized zirconium implants in this study do not demonstrate the improved clinical outcomes expected following the promising knee simulator studies. In this retrospective study, a review of clinical and radiological outcomes concludes that femoral components fabricated from both materials do not have differing clinical effectiveness.

After a preliminary review of patient demographics, as well as clinical and radiographic outcomes, a subgroup within the $\mathrm{CoCr}$ group was established in order to more directly compare clinical effectiveness between cohorts of similar age. It could be argued that since the oxidized zirconium components were implanted in younger and more active patients, they were subjected to a more rigorous in vivo environment. However, upon comparison to a patient cohort of a similar age range, no significant statistical or clinical differences were found among patient cohorts.

Similar preoperative KSS were noted for both groups, as well as the CoCrMo alloy subgroup with an age less than 65 years. Postoperative mean KSS were also similar $(P=0.13031$ and 0.8139 , resp.). It was hypothesized that oxidized zirconium would demonstrate better clinical outcomes, including improved knee scores and range of motion; however, such was not the case in the present study. Previously published studies have found similar results, including that of Kim et al. which reported no significant difference in knee scores between a single TKA design with oxidized zirconium and CoCrMo alloy femoral components implanted in patients with an average followup of 7.5 years [14]. A power analysis showed that this study had $100 \%$ power to detect a KSS difference of 5; hence, it is reasonable to conclude that there was no difference between the two groups based on KSS.

In addition to KSS, maximum flexion was measured at each most recent followup. Comparing the measurements taken at the most recent follow-up visit, the values showed a difference of 2.7 degrees $(P=0.0436)$. Though the difference between the two values was considered statistically significant, there is no clinical significance because two to three degrees of flexion are within the expected measurement error, and even if a true difference is likely inconsequential for patient recovery. This analysis of maximum flexion corroborates other clinical research interpretations [14]. Analysis of the CoCrMo alloy subgroup and the oxidized zirconium showed no statistical difference when comparing flexion $(P$ value $=0.3584)$. The results from these tests correlate with the clinical presentation and with other clinical studies $[13,14]$ showing no significant difference in relation to range of motion between patients who received the oxidized zirconium compared to the CoCrMo alloy.

Manifestations of metal allergy, mainly to nickel, in TKA are not well defined, and there are little publications on patient presentation of a metal allergy. Gao et al. and Thomas et al. noted that metal allergies can present as dermatitis, swelling, and pain during the first three months after surgery, and continuing for years afterward [17, 18]. Following the matrix of symptoms including swelling, dermatitis, pain, prolonged use of medications for pain, and flexion less than 115 degrees, patients in the present study were investigated for having a reasonable probability of metal allergy. Of the patients that experienced pain, only one patient experienced at least three of the five clinical presentations of a metal allergy. This patient was implanted with an oxidized zirconium femoral component and experienced pain throughout his/her follow-up period ( 3 years) with no sign of infection, implant loosening or malalignment. A conclusion cannot be drawn from the current study data set concerning metal allergy due to small sample size and only one possible occurrence.

When the twelve patients who experienced pain at the most recent followup were compared, no statistical difference was calculated between the two materials $(P$ value $=0.2388$ ) Comparison of the CoCrMo alloy main group and subgroup to the oxidized zirconium group shows no difference between pain experienced by patients in these groups $(P=1)$.

When considering the clinical and radiological presentations of both types of femoral components, the results show no significant differences in outcomes between the oxidized zirconium and CoCrMo alloy cohorts. Reviewing data containing Knee Society Scores, knee flexion, probability of a metal allergy, presentations of pain and other complications, and number of revisions demonstrates that there are no statistical or clinical differences in values obtained from both cohorts. This also holds true with respect to radiographic outcomes, in which neither patient group presented with osteolysis nor radiolucencies is directly associated with femoral component material or failure. Summation of this average 5.6-year in vivo study does not corroborate with the improved clinical outcomes the knee simulator studies suggest for the benefits of oxidized zirconium over CoCrMo alloy. Our statistical analysis and data provide similar data to the clinical studies reporting on the Genesis II (Smith \& Nephew, Memphis, TN, USA) TKA, which showed similar early clinical outcomes between femoral components fabricated from the two materials of interest $[10,13,14]$. 
ISRN Biomaterials

The cost comparison component of this study shows a substantial difference in price between the oxidized zirconium and the CoCrMo alloy femoral components. At the time of implantation, the hospital direct implant cost of the total knee implant system with an oxidized zirconium femur was $\$ 1,900$ (55\%) more expensive than that of the same TKA with a CoCrMo femoral component.

With no apparent added clinical benefit of the oxidized zirconium components compared to the CoCrMo alloy components, the extra costs of oxidized zirconium may not be warranted. Other medium-term studies focusing on the clinical outcomes of patients with oxidized zirconium and CoCrMo alloy TKAs have also shown similar results and that patients do not have a preference for one implant over the other in bilateral TKA series with each knee receiving implants differing only by the femoral oxidized zirconium versus CoCrMo components [13].

There are some inherent limitations of this retrospective review of clinical and radiographic outcomes. The number of patients in each patient cohort may not be sufficient to appreciate a potentially very small incidence of adverse events. Additionally, the statistically significant age difference between cohorts posed a challenge when comparing clinical and radiographic outcomes. This challenge was addressed by creating a subgroup of one cohort with a similar age range as the oxidized zirconium cohort in order to effectively compare two groups with hypothetically similar activity levels. The average follow-up time of both patient cohorts of approximately 5-6 years may not be sufficient to fully capture potential adverse events that occur at more longterm followup. However, wear-induced osteolysis can present radiographically within the first 3 postoperative years [19] and metal allergy typically presents within the first 3 months, potentially lasting until revision surgery is performed $[17,18]$. Therefore, patients with a minimum of one year of followup were included in the current patient cohorts.

\section{Conclusion}

Previous in vitro wear analysis of oxidized zirconium versus CoCrMo showed an improved wear-resistant bearing surface and decreased metal ion release. This suggests that oxidized zirconium implants were likely to have a more favorable clinical outcome compared to traditional cobalt chromium implants. The present study of the two biomaterials took into account Knee Society Scores, knee flexion, incidence of a metal allergy, pain, and other reported complications. Both pre- and postoperative KSS and pain scores had no statistical variation between the two component materials. There was no clinical difference in knee flexion, number of patients experiencing pain, complications, or radiographic findings of implant related osteolysis. Long-term follow-up studies are required to conduct a true cost analysis comparison between these two biomaterials. However, considering that the hospital purchase cost for the oxidized zirconium implant at the institution where these surgeries were performed was \$1900 (55\%) more than the CoCrMo alloy implant, and with no added clinical benefit at early followup, the oxidized zirconium implant may not worth the additional cost.

\section{Conflict of Interests}

The authors declare that there is no conflict of interests regarding the publication of this paper.

\section{Acknowledgments}

Ms. Sandy Fowler and Mrs. Katharine A. Pace are acknowledged for their contributions to the paper and data preparation.

\section{References}

[1] S. Kurtz, K. Ong, E. Lau, F. Mowat, and M. Halpern, "Projections of primary and revision hip and knee arthroplasty in the United States from 2005 to 2030," The Journal of Bone and Joint Surgery A, vol. 89, no. 4, pp. 780-785, 2007.

[2] M. Spector, M. D. Ries, R. B. Bourne, W. S. Sauer, M. Long, and G. Hunter, "Wear performance of ultra-high molecular weight polyethylene, on oxidized zirconium total knee femoral components," The Journal of Bone and Joint Surgery A, vol. 83, no. 2, pp. S80-S86, 2001.

[3] J. A. Davidson, C. M. Asgian, A. K. Mishra, and P. Kovacs, "Zirconia $\left(\mathrm{ZrO}_{2}\right)$-coated zirconium-2.5 Nb alloy for prosthetic knee bearing applications," Bioceramics, vol. 5, pp. 389-401, 1992.

[4] M. C. Galetz, E. W. Fleischmann, C. H. Konrad, A. Schuetz, and U. Glatzel, "Abrasion resistance of oxidized zirconium in comparison with CoCrMo and titanium nitride coatings for artificial knee joints," Journal of Biomedical Materials Research $B$, vol. 93, no. 1, pp. 244-251, 2010.

[5] M. C. Galetz, S. H. Seiferth, B. Theile, and U. Glatzel, "Potential for adhesive wear in friction couples of UHMWPE running against oxidized zirconium, titanium nitride coatings, and cobalt-chromium alloys," Journal of Biomedical Materials Research B, vol. 93, no. 2, pp. 468-475, 2010.

[6] J. D. DesJardins, B. Burnikel, and M. LaBerge, "UHMWPE wear against roughened oxidized zirconium and $\mathrm{CoCr}$ femoral knee components during force-controlled simulation," Wear, vol. 264, no. 3-4, pp. 245-256, 2008.

[7] T. J. Heyse, D. X. Chen, N. Kelly, F. Boettner, T. M. Wright, and S. B. Haas, "Matched-pair total knee arthroplasty retrieval analysis: oxidized zirconium versus CoCrMo," The Knee, vol. 18, no. 6, pp. 448-452, 2011.

[8] M. D. Ries, A. Salehi, K. Widding, and G. Hunter, "Polyethylene wear performance of oxidized zirconium and cobalt-chromium knee components under abrasive conditions," The Journal of Bone and Joint Surgery A, vol. 84, no. 2, pp. 129-135, 2002.

[9] S. E. White, L. A. Whiteside, D. S. McCarthy, M. Anthony, and R. A. Poggie, "Simulated knee wear with cobalt chromium and oxidized zirconium knee femoral components," Clinical Orthopaedics and Related Research, no. 309, pp. 176-184, 1994.

[10] R. S. Laskin, "An oxidized Zr ceramic surfaced femoral component for total knee arthroplasty," Clinical Orthopaedics and Related Research, no. 416, pp. 191-196, 2003.

[11] J. M. Brandt, L. Guenther, S. O’Brien, A. Vecherya, T. R. Turgeon, and E. R. Bohm, "Performance assessment of femoral knee components made from cobalt-chromium alloy and oxidized zirconium," The Knee, vol. 20, no. 6, pp. 388-396, 2013. 
[12] M. Innocenti, R. Civinini, C. Carulli, F. Matassi, and M. Villano, "The 5-year results of an oxidized zirconium femoral component for TKA," Clinical Orthopaedics and Related Research, vol. 468, no. 5, pp. 1258-1263, 2010.

[13] C. Hui, L. Salmon, S. Maeno, J. Roe, W. Walsh, and L. Pinczewski, "Five-year comparison of oxidized zirconium and cobalt-chromium femoral components in total knee arthroplasty a randomized controlled trial," The Journal of Bone and Joint Surgery A, vol. 93, no. 7, pp. 624-630, 2011.

[14] Y. H. Kim, J. W. Park, and J. S. Kim, "Comparison of the genesis II total knee replacement with oxidised zirconium and cobalt-chromium femoral components in the same patients: a Prospective, Double-blind, Randomized Controlled Study," The Journal of Bone and Join Surgery, vol. 94, no. 9, pp. 1-7, 2012.

[15] M. C. Inacio, G. Cafri, E. W. Paxton, S. M. Kurtz, and R. S. Namba, "Alternative bearings in total knee arthroplasty: risk of early revision compared to traditional bearings: an analysis of 62, 177 primary cases," Acta Orthopaedica, vol. 84, no. 2, pp. 145$152,2013$.

[16] J. N. Insall, L. D. Dorr, R. D. Scott, and W. N. Scott, "Rationale of the knee society clinical rating system," Clinical Orthopaedics and Related Research, no. 248, pp. 13-14, 1989.

[17] X. Gao, R.-X. He, S.-G. Yan, and L.-D. Wu, "Dermatitis associated with chromium following total knee arthroplasty," The Journal of Arthroplasty, vol. 26, no. 4, pp. 665.e13-665.e16, 2011.

[18] P. Thomas, M. Thomas, B. Summer et al., "Impaired woundhealing, local eczema, and chronic inflammation following titanium osteosynthesis in a nickel and cobalt-allergic patient," The Journal of Bone and Joint Surgery A, vol. 93, no. 11, article e61, pp. 1-5, 2011.

[19] S. K. Gupta, A. Chu, A. S. Ranawat, J. Slamin, and C. S. Ranawat, "Review article: osteolysis after total knee arthroplasty," The Journal of Arthroplasty, vol. 22, no. 6, pp. 787-799, 2007. 

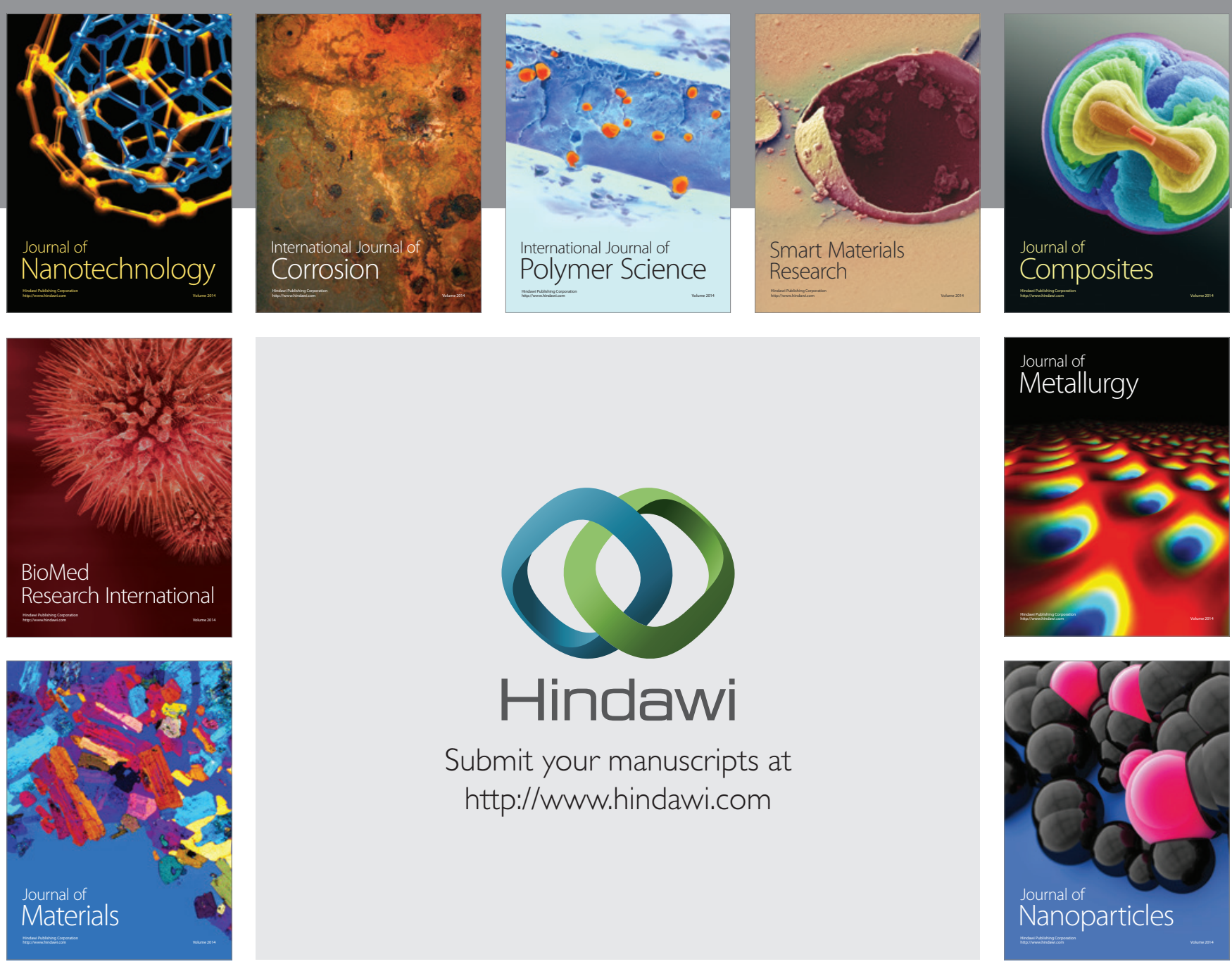

Submit your manuscripts at http://www.hindawi.com
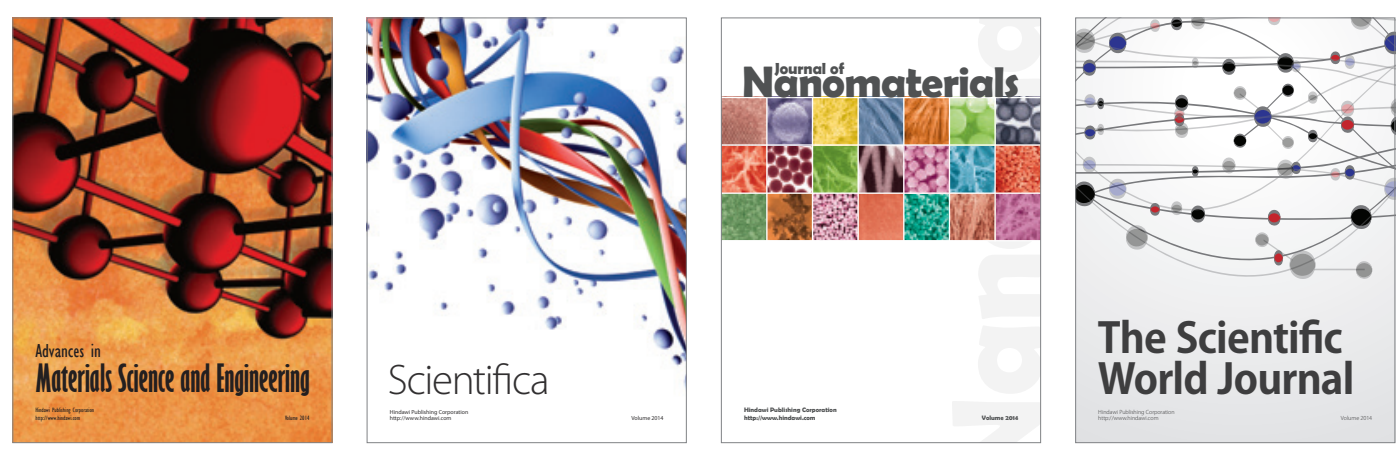

\section{The Scientific World Journal}
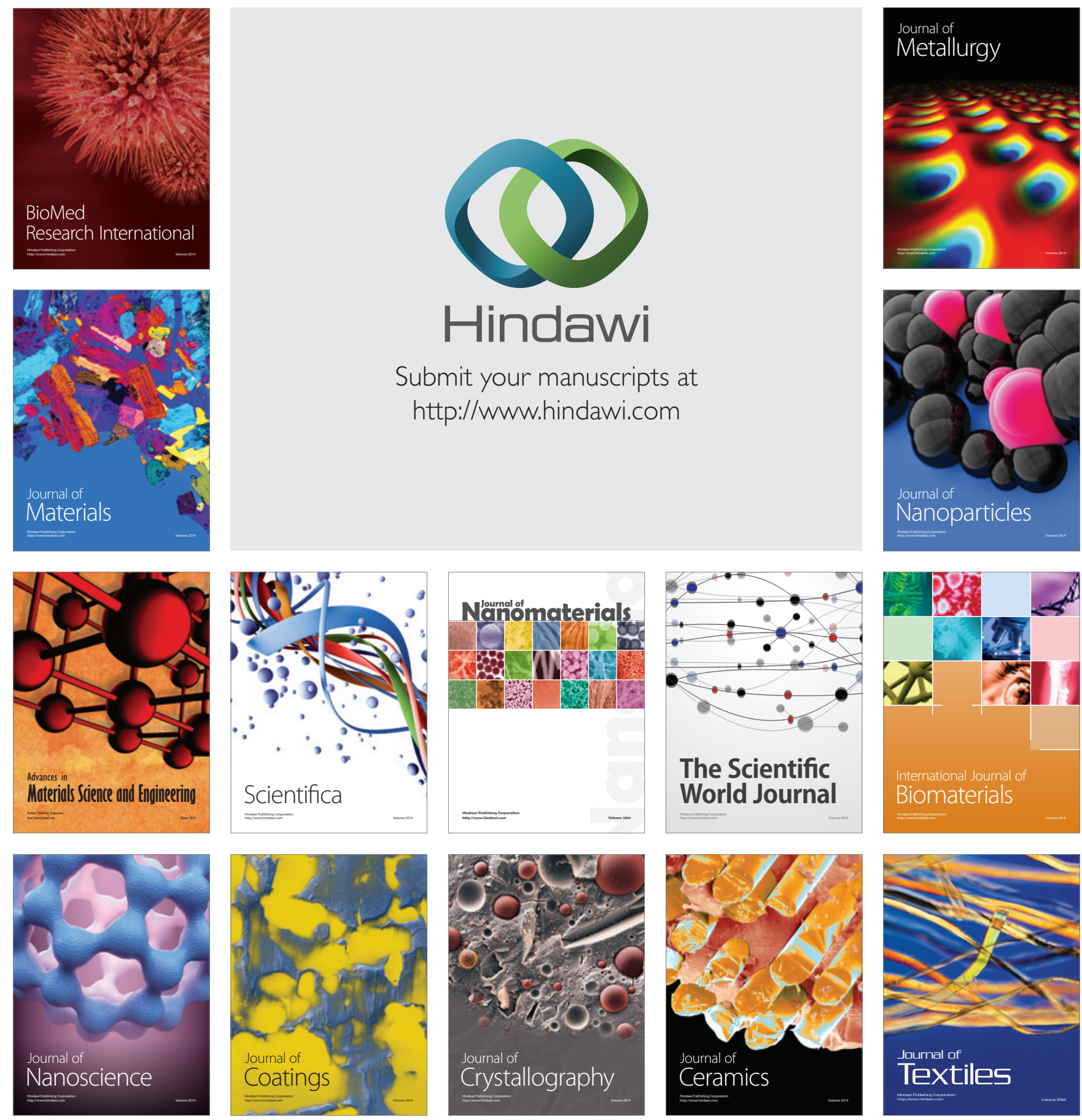